Zinc-finger nucleases meet iPS cells

\section{Zinc positive: tailored genome engineering meets reprogramming}

$\mathrm{T}$ Cathomen and A Schambach

Gene Therapy (2010) 17, 1-3; doi:10.1038/gt.2009.151; published online 12 November 2009

Is it a dream coming true for gene and cell therapy? Maybe not quite yet, but two recent reports ${ }^{1,2}$ describing targeted genome engineering in pluripotent stem cells certainly bring us a big step closer to the dream of finding a rational cure for patients suffering from various inherited and acquired disorders. The foremost goal of human cell-based therapy has been to develop safe and predictable technologies that allow treatment of patients with inherited, acquired or degenerative disorders by providing a ready supply of highly defined transplantable cells. Because of their plasticity and capacity for self-renewal, induced pluripotent stem cells (iPSCs) are a promising and almost unlimited source for personalized cell-based therapies. The ability to genetically modify iPSCs ad libitum now extends the possible operation range of stem cell therapies from regenerative medicine to human gene therapy.

The successful implementation of this vision was based on productive collaborations between stem cell and genome engineering laboratories, and is the culmination of incremental technical advances that have been achieved in the respective fields in the last few years. Three years ago, Takahashi and Yamanaka ${ }^{3}$ reported the seminal finding that iPSCs can be generated from somatic mouse fibroblasts by over-expression of just four transcription factors (Oct4, Sox2, Klf4 and c-Myc). Most likely, this cocktail activates a network of transcription factors that induce epigenetic changes and 'reprogram' the somatic cells into a pluripotent state. As a result, iPSCs have similar properties as embryonic stem cells (ESCs). The finding that human cells can be reprogrammed using the same strategy $\mathrm{y}^{4}$ has opened the door for new approaches in regenerative medicine, such as the generation of patient-specific iPSCs ${ }^{5}$ that will avoid the ethical dilemma of using early pre-implantation embryos for the generation of pluripotent stem lines. It is noteworthy that the portfolio of techniques to generate iPSCs has clearly improved and now includes various integrating and non-integrating vector strategies as well as small-molecule drugs. ${ }^{6} \mathrm{Hu}-$ man iPSCs, just like ESCs, can be cultured as clonal lines almost indefinitely and can be differentiated into the mature tissues of all three germ layers.

So, what are the tools to introduce specific changes into the genome of patient-derived iPSCs for therapeutic benefit? A very promising approach is based on the zinc-finger nuclease (ZFN) technology. A ZFN subunit consists of a non-specific endonuclease domain fused to a specific DNA-binding domain composed of engineered zinc-finger motifs that tether the nuclease domain to a preselected chromosomal site. Upon dimerization of two ZFN subunits at the target site, the $\mathrm{ZFN}$ pair specifically cleaves the DNA to trigger the ensuing genome editing (Figure 1). ${ }^{7,8}$ If an appropriate donor DNA with homology to the target locus is provided, the DNA damage can be repaired by homologous recombination, which, in the reports discussed here, prompted the sitespecific integration of an expression cassette at the site of the DNA break. ZFN-mediated genome engineering has been achieved in a wide variety of cell types and organisms, including human ESCs. ${ }^{9}$ The foremost parameters that were fundamental to the successful implementation of the ZFN technology include re-engineering of the catalytic nuclease activity and the ability to produce highly specific DNA-binding do- mains. ${ }^{7,8}$ Both are critical to ensure high on-target activity combined with low genotoxicity due to offtarget activity. Although similar nuclease domains were used in the two reports under discussion, the manner in which the DNA-binding domains were produced was quite dissimilar. Zou et al. ${ }^{1}$ used a publicly available technology developed by the Zinc Finger Consortium (www.zincfingers.org), whereas Hockemeyer et al. ${ }^{2}$ relied on a proprietary platform developed by Sangamo BioSciences (www.sangamo. com). The question of which zincfinger engineering platform works better is a moot point-both approaches rendered $\mathrm{ZFNs}$ that mediated the introduction of defined genetic modifications in highly sensitive iPSCs, without reported side effects that would affect the pluripotency of these cells or their genetic stability.

Jaenisch's team used the ZFNs in classical knock-in approaches and demonstrated the versatility of the technology. ${ }^{2}$ They generated reporter iPSC lines by knocking-in an EGFP marker gene under the endogenous control of the OCT4 promoter, hence producing a pluripotency indicator allele. Furthermore, they established that targeted integration into the $A A V S 1$ locus (the prototypical integration site for adeno-associated virus type 2) allows for robust transgene expression (probably similar to the better-known ROSA26 locus $\left.{ }^{10}\right)$. Finally, they showed that targeting of a silent locus (PITX3, encoding a transcription factor that is selectively expressed in the midbrain) is possible. The latter is not trivial and ZFN-mediated gene targeting in closed chromatin areas is not expected to work in all instances. Importantly, in all cases a puro ${ }^{R}$ selection cassette was co-introduced, which allowed the authors to select for puromycin-resistant iPSC clones. The targeting efficiency ranged between 8 and $96 \%$, depending on the target locus, the donor architecture and the ZFN pair used. Zou et al. ${ }^{1}$ targeted an artificial EGFP locus and the endogenous PIG- $A$ locus, which is mutated in patients suffering from X-linked paroxysmal nocturnal hemoglobinuria, a complement-induced hemolytic form of 

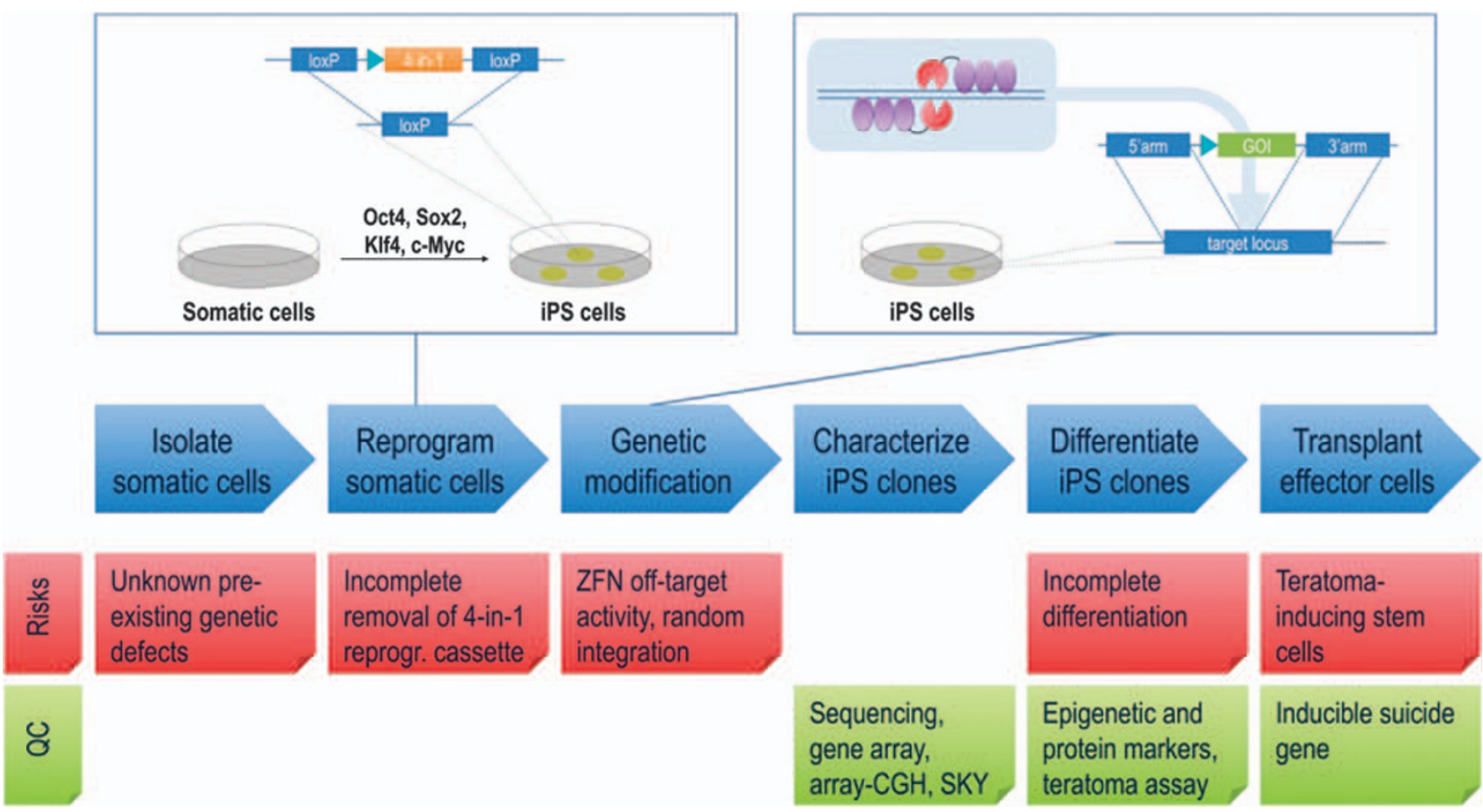

Figure 1 Roadmap to a therapy with patient-specific iPSCs. (1) Suitable somatic cells are isolated from the patient. (2) A '4-in-1' reprogramming cassette is transferred into these somatic cells to generate iPSCs (refer the text for details). After reprogramming, the '4-in-1' cassette is excised using, for example, the Cre/loxP system. (3) A donor DNA containing a gene of interest (GOI) flanked by homology arms to the target locus $\left(5^{\prime} \mathrm{arm} / 3^{\prime} \mathrm{arm}\right)$ is used as a substrate for gene targeting. The frequency of gene targeting is enhanced by about 1000 -fold on inserting a DNA cut in the target locus with tailored ZFNs. (4) After antibiotic selection, the iPSC clones are expanded and thoroughly characterized. A full characterization should include the confirmation of the pluripotent state of the cells, a genome analysis that comprises deep sequencing and an evaluation of the chromosomal integrity, for example, by microarray-based comparative genomic hybridization (array-CGH) and/or spectral karyotyping (SKY), and an assessment of the transcriptome integrity by gene array. (5) The genetically modified iPSCs that pass this quality control (QC) are differentiated into the desired effector cells. Epigenetic and/or protein markers should be used to verify the differentiation state. (6) Fully differentiated cells are transplanted into patients. An inducible suicide gene cassette can be included as an additional safety measurement. The blue triangle indicates the promoter.

anemia. In the latter case, they used a hygro ${ }^{R}$-containing targeting cassette and achieved mono-allelic disruption of the PIG-A locus in $45 \%$ of hygromycin-resistant male iPSC clones.

For both reports, antibiotic selection was instrumental as only about 1 in 3500 iPSCs contained an integrated form of the donor DNA. However, on average $\approx 50 \%$ of antibiotic-resistant clones harbored the desired genetic modification, which is much higher than the percentage observed for conventional gene targeting in murine ESCs. As previously reported for other cells, the average stimulation of gene targeting by ZFNs in human iPSCs was about 1000-fold. All these numbers demonstrate the extraordinary potential of ZFN-mediated genome engineering in human iPSCs. At present, this technology will prove useful in generating novel genetic tools to study the molecular basis of inherited disorders or cell fate decisions, as well as cell type-specific reporter systems to improve differentiation protocols. In the future, precise genome engineering in patient-derived iPSCs will open the door to personalized cell replacement therapy. However, a few steps have to be taken and hurdles (Figure 1) overcome to make this technology available at the bedside:

1. Isolation of suitable and easily obtainable cells from patients (for example, fibroblasts, keratinocytes, hematopoietic cells or banked umbilical cord blood cells).

2. Genetic reprogramming into iPSCs using excisable or transient/non-integrating reprogramming cassettes.

3. Genetic modification using custom-designed ZFNs. Owing to the low absolute gene targeting frequencies in human iPSCs $(\approx 1$ in 8000 cells), correction of an inborn mutation in situ in the absence of any selection is not feasible at the moment. However, targeted addition of a partial cDNA in an upstream exon or targeted addi- tion of a whole transgene cassette into a 'safe harbor' are valuable alternatives (for details, see Cathomen and Joung ${ }^{8}$ ). It remains to be demonstrated whether the AAVS1 locus presents such a 'safe harbor'. Also, ZFN-treated cells have to be thoroughly examined for nuclease-induced DNA damage.

4. Characterization of iPSC clones. The fact that individual iPSC clones can be expanded almost without limitation offers the chance to fully characterize these clones. The extent of functional and genetic characterization of iPSC clones suitable for medical use remains to be defined.

5. Safe and efficient differentiation into multipotent (for example, hematopoietic stem cells) or mature effector cells (for example, leukocytes, erythrocytes). Here, the testing for remaining cells capable of initiating teratoma formation is critical.

6. Transplantation (and engraftment) of genetically modified 
cells with potency assays in preclinical disease models and the possibility of conditional elimination of progeny cells (suicide gene).

In conclusion, the two reports ${ }^{1,2}$ suggest that ZFN-mediated tailored genome modifications can be effectively and meaningfully applied to human iPSCs. For future therapeutic applications, the resulting cells have to be monitored carefully for unintended genetic modification and for the full differentiation state of the desired effector cells. Although these reports may herald the dawn of a new chapter in combined gene/cell therapy, further improvements in the upstream technologies (genome engineering and reprogramming), together with strongly enhanced downstream technologies (differentiation and transplantation), will be important for eventual clinical application of patient-specific iPSCs.

\section{Conflict of interest}

The authors declare no conflict of interest.

Drs $T$ Cathomen and A Schambach are at the Department of Experimental Hematology, Hannover Medical School, Carl-NeubergStrasse 1, Hannover D-30625, Germany.

E-mail:cathomen.toni@mh-hannover.de

1 Zou J, Maeder ML, Mali P, Pruett-Miller SM, Thibodeau-Beganny S, Chou BK et al. Gene targeting of a disease-related gene in human induced pluripotent stem and embryonic stem cells. Cell Stem Cell 2009; 5: 97-110.

2 Hockemeyer D, Soldner F, Beard C, Gao Q, Mitalipova M, DeKelver RC et al. Efficient targeting of expressed and silent genes in human ESCs and iPSCs using zinc-finger nucleases. Nat Biotechnol 2009; 27: 851-857.

3 Takahashi K, Yamanaka S. Induction of pluripotent stem cells from mouse embryonic and adult fibroblast cultures by defined factors. Cell 2006; 126: 663-676.
$4 \mathrm{Yu}$ J, Vodyanik MA, Smuga-Otto K, Antosiewicz-Bourget J, Frane JL, Tian S et al. Induced pluripotent stem cell lines derived from human somatic cells. Science 2007; 318: 1917-1920.

5 Park IH, Arora N, Huo H, Maherali N, Ahfeldt T, Shimamura A et al. Diseasespecific induced pluripotent stem cells. Cell 2008; 134: 877-886.

6 Baker M. Stem cells: fast and furious. Nature 2009; 458: 962-965.

7 Carroll D. Progress and prospects: zincfinger nucleases as gene therapy agents. Gene Therapy 2008; 15: 1463-1468.

8 Cathomen T, Joung KJ. Zinc-finger nucleases: the next generation emerges. Mol Therapy 2008; 16: 1200-1207.

9 Lombardo A, Genovese P, Beausejour CM, Colleoni S, Lee YL, Kim KA et al. Gene editing in human stem cells using zinc finger nucleases and integrasedefective lentiviral vector delivery. Nat Biotechnol 2007; 25: 1298-1306.

10 Irion S, Luche H, Gadue P, Fehling HJ, Kennedy M, Keller G. Identification and targeting of the ROSA26 locus in human embryonic stem cells. Nat Biotech 2007; 25: $1477-1482$. 